Voici un siècle, la découverte de la supraconductivité fut le fruit d'une politique déterminée des Pays-Bas de soutien à la recherche et l'enseignement pour maintenir leur rang, et de l'obstination d'un élève de Van der Waals, Heike Kamerlingh Onnes, qui comprit que pour aller plus bas vers le zéro absolu de température, il fallait désormais mettre en œuvre des moyens industriels.

Bientôt, avec la nouvelle mécanique quantique, la supraconductivité suscita l'intérêt de la jeune génération de théoriciens. En 1933, Meissner et Ochsenfeld mettaient en évidence l'expulsion complète du champ magnétique du volume d'un supraconducteur. Pour les frères London, cette expulsion était un diamagnétisme parfait : le concept d'état macroscopique quantique venait d'être inventé.

\title{
Faute d'entrer par la bonne porte : les jeunes et longues années de la supraconductivité (1911-1940)
}

Georges Waysand $^{(1)}$ (waysand@orange.fr), Christian Joas ${ }^{(2)}$ et Jean Matricon ${ }^{(3)}$

(1) Laboratoire Souterrain Bas Bruit, UMS 2202 UNSA/CNRS/OCA, La Grande Combe, 84400 Rustrel

(2) Fritz-Haber Institut des MPG et Max-Planck Institut für Wissenschaftgeschichte, Boltzmannstr. 22, 14195 Berlin, Allemagne (3) Université Denis Diderot Paris 7

Le 8 avril 1911, au Laboratoire des Basses Températures de Leiden, depuis trois ans le seul endroit au monde à liquéfier de l'hélium, Gilles Holst observe une disparition brutale de la résistivité d'un capillaire de mercure à $4,19 \mathrm{~K}$ en présence de Kamerlingh Onnes. À la différence de celle de la liquéfaction de l'hélium trois ans plus tôt, cette naissance ne fut pas célébrée en fanfare. Pendant de nombreuses années, ce résultat surprenant resta interprété comme le cas limite de la conductivité métallique ordinaire au voisinage du zéro absolu. En témoigne la participation de Kamerlingh Onnes à l'événement image d'Épinal de la physique moderne que fut la première conférence Solvay en 1911, pour faire le point sur «la théorie cinétique de la matière et la théorie quantique du rayonnement ».

\section{Un centenaire peut en cacher un autre}

Le 2 novembre 1911, après un exposé de Walther Nernst sur les chaleurs spécifiques à basses températures, Kamerlingh Onnes présenta ses résultats "Sur les résistances électriques ", qui montraient tous des résistivités décroissantes aux basses températures, contrairement à certaines attentes initiales fondées sur la notion de gel des électrons. Parmi les métaux, le choix du mercure, facile à obtenir très pur, s'était imposé. Mais pour mesurer sa résistivité, le montage n'avait rien de trivial : il avait fallu nécessairement travailler sur des capillaires pour avoir des résistances encore décelables à basse température et, de plus, assurer des prises de contact qui n'engendrent pas d'effet thermoélectrique trop important. Kamerlingh Onnes qualifia la variation observée en dessous de 4,2 $\mathrm{K}$ de « diminution très rapide et presque une discontinuité ". Son exposé ne suscita qu'une seule question. Pensant à une transition de phase engendrant une contraction qui augmenterait la densité d'électrons libres, Paul Langevin demanda "si la variation très rapide qui se produit au voisinage de $4^{\circ}$ absolus [...] ne correspond pas à un changement d'état s'accompagnant par exemple d'une variation brusque de volume ». Kamerlingh Onnes répondit par un véritable programme pluriannuel d'expériences, avant de conclure : "En tout cas, le nouvel état du mercure se distingue de celui au-dessus de $4^{\circ}, 2 \mathrm{~K}$ par une conductibilité tout à fait extraordinaire. " On a connu des naissances plus triomphales. Pour tous les présents, c'était la conductivité parfaite au voisinage du zéro absolu qui était à comprendre. Ainsi, la première manifestation connue de la supraconductivité allait être prise pendant un quart de siècle pour sa propriété caractéristique.

Décembre 1912 apporta une autre surprise : la découverte de la supraconductivité de l'étain et, dans la foulée, celle du plomb. Kamerlingh Onnes, entrepreneur dans l'âme, y vit d'emblée la possibilité de réaliser des aimants sans fer... espoir vite déçu. La faible valeur de la densité de courant critique ramenait les bobines de fil de plomb à l'état résistif, ce qui fut interprété à tort comme un effet analogue au chauffage du conducteur. Ce n'est qu'en 1916 que Francis Briggs Silsbee émit l'hypothèse que le courant critique crée à la surface du conducteur le champ magnétique critique. Après son prix Nobel de 1913 "pour la préparation de l'hélium liquide ", et non pour la supraconductivité, Kamerlingh Onnes vit couronnées de succès les premières expériences de courant permanent lancé par induction dans une bobine d'un millier de tours de fil de plomb. 

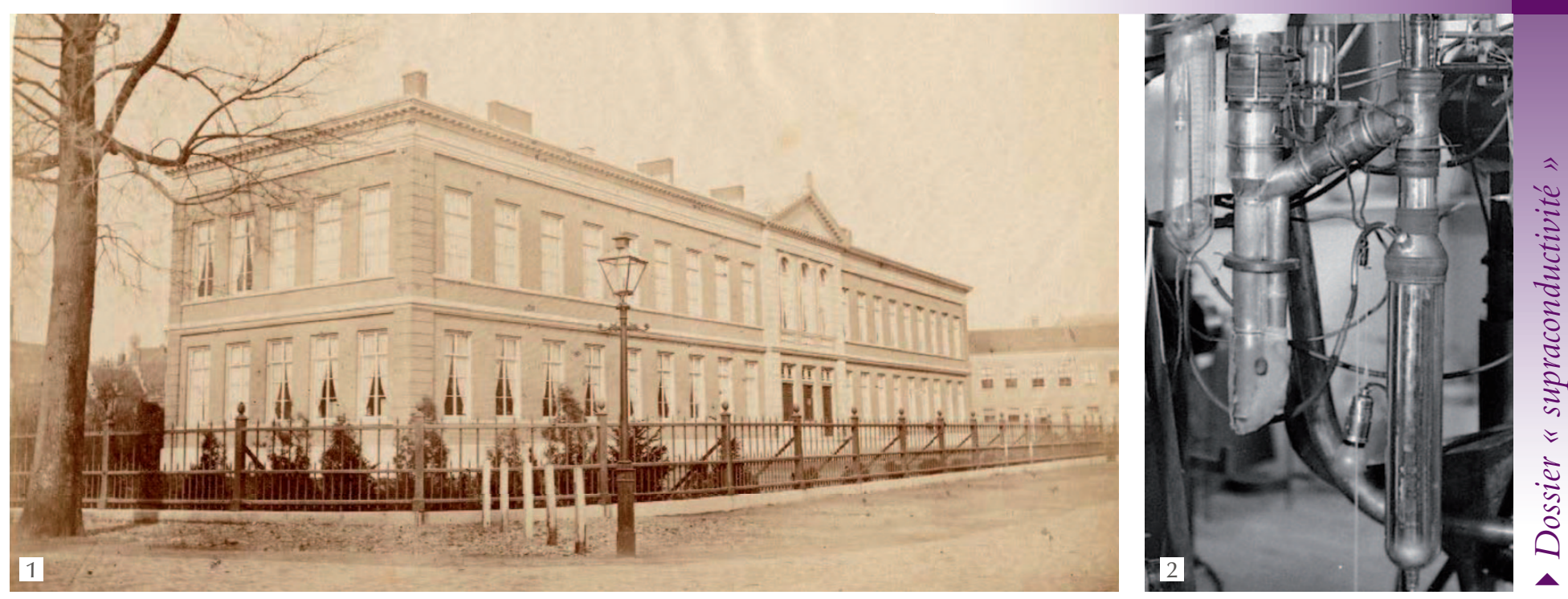

1. Le laboratoire de physique, chimie, anatomie et physiologie de Leiden, vers 1880. 2. Le dispositif de l'expérience du 8 avril 1911.

(Courtoisie : Peter Kes, Kamerlingh Onnes Laboratory, Leiden University.)

Une autre expérience de Kamerlingh Onnes avant le début de la Première Guerre mondiale eut une importance capitale pour la première vague de tentatives théoriques d'expliquer la supraconductivité : l'absence d'effet Hall dans l'état supraconducteur conforta l'idée qu'on avait affaire à des trajectoires électroniques spécifiques, véritables canaux sans collisions.

\section{Premières tentatives théoriques}

Frederick Lindemann, élève de Nernst, rentré en Angleterre dès le début des hostilités, fut écarté de l'effort de guerre. Il continua donc ses recherches et avança en 1915 qu'aux basses températures, un métal était composé de deux réseaux cristallins de paramètres identiques et emboîtés l'un dans l'autre, l'un pour les ions, l'autre pour les électrons, ce dernier pouvant coulisser à travers le cristal d'ions sans dissiper aucune énergie. Le modèle d'atome proposé par Niels Bohr en 1913 fournissait la possibilité de concrétiser l'idée du cristal d'électrons. Son utilisation pour la conduction métallique fut faite indépendamment par Carl Benedicks (en Suède), Percy Bridgman (aux États-Unis) en 1916-1917 et Fritz Haber (en Allemagne) en 1919. Pour ce dernier, «le point de vue qui vient naturellement à l'esprit est de concevoir la supraconductivité comme un état dans lequel les électrons de valence d'un métal se déplacent sur des orbites tangentes en des points d'égale vitesse ". C'était reprendre une idée de Johannes Stark pour concilier bonne conductivité électrique et faible contribution des électrons à la chaleur spécifique aux basses températures. La conductivité électrique parfaite était possible au zéro absolu.
Pendant le conflit, Einstein, en dépit de ses souhaits, n'avait pu quitter l'atmosphère nationaliste de Berlin pour séjourner à Leiden à l'invitation de son ami Ehrenfest. Les obstacles à cette visite ne furent levés qu'en 1919. Einstein devint professeur invité de 1920 à 1923, ce qui impliquait des séjours de plusieurs semaines. Il put ainsi participer aux nombreuses discussions qui se tenaient à Leiden sur la supraconductivité. En 1922, pour le $40^{\mathrm{e}}$ anniversaire de la prise de chaire de Kamerlingh Onnes, Einstein résuma l'état de la question «Tout se passe comme si, en l'état actuel de nos connaissances, les électrons libres dans un métal n'existent pas du tout... il semble inévitable que les supercourants soient portés par des chaînes moléculaires closes sur elles-mêmes. » Ce qu'il pondérera immédiatement. "Avec notre abyssale ignorance de la mécanique quantique des systèmes composés, nous sommes très loin d'être capables de composer une théorie à partir de ces vagues idées. »

\section{La nouvelle mécanique quantique et la deuxième vague de tentatives théoriques}

Au milieu des années 20, les électrons dans les métaux étaient un banc d'essai pour la mécanique quantique. Après l'utilisation par Pauli en 1926, puis Sommerfeld en 1927, de la statistique de Fermi-Dirac pour un gaz d'électrons libres, Felix Bloch en 1928 fournit la solution au problème des électrons dans un cristal métallique que lui avait proposé d'étudier Werner Heisenberg. Le réseau ionique y est approximé par un potentiel périodique à trois dimensions ; les interactions entre électrons sont négligées. Reprenant l'hypothèse mise en œuvre par Heitler et London pour calculer la liaison entre atomes dans la molécule d'hydrogène - acte de naissance de la chimie quantique - Félix Bloch partait d'une base d'orbitales non perturbées d'atomes isolés. À peu près simultanément, Werner Heisenberg, reprenant lui aussi la méthode de Heitler-London, montrait que le ferromagnétisme est parfaitement descriptible en termes d'interactions entre spins électroniques parallèles. L'intégrale d'échange expliquait le champ moléculaire de Pierre Weiss, tout comme elle avait expliqué la liaison des atomes de la molécule d'hydrogène.

Ce succès va donner naissance à un nouveau paradigme. Tous ceux qui vont s'intéresser à la supraconductivité voudront désormais la traiter comme le ferromagnétisme. Entre 1929 et 1933, plus d'une douzaine de théoriciens vont s'attaquer à la supra : Bohr, Pauli, Bloch, Heisenberg, Landau, Brillouin, Frenkel... allant même parfois jusqu'à reprendre l'idée d'un cristal d'électrons. Tous ces modèles étaient fondés sur la conviction qu'une théorie de la supraconductivité devait proposer un mécanisme par lequel, en l'absence de tout champ extérieur, un métal devait être le siège d'un courant... tout comme un ferromagnétique a une aimantation permanente sans champ extérieur. Felix Bloch en privé, puis indépendamment Léon Brillouin en mai 1935 au meeting de la Royal Society évoqué plus loin, établirent que l'état d'énergie minimum était un état de courant nul. La démonstration de Brillouin est élémentaire

«Supposons qu'un courant I circule dans un métal (qu'il soit de surface ou en volume). L'énergie du métal sera E ; nous voulons prouver que E ne peut être minimum. 


\section{>>}

Si pendant un court intervalle de temps dt nous appliquons une différence de potentiel $P$ entre les deux extrémités du conducteur, l'énergie de celui-ci sera augmentée d'une quantité P. I. dt ; en changeant le signe de $\mathrm{P}$ on peut rendre cette quantité positive ou négative, donc nous voyons qu'il y a toujours une possibilité de réduire l'énergie E, qui ne peut donc être minimale ».

Ce premier théorème fut accompagné par Bloch, rapporteur de tous les papiers sur la supraconductivité soumis au Zeitschrift, d'un deuxième qui n'était qu'une boutade : " toute théorie de la supraconductivité est a priori une théorie fausse. »

\section{Expériences hors contexte et expériences décisives}

Deux expériences furent immédiatement reconnues comme décisives et précipitèrent l'évolution de la compréhension de la supraconductivité, malgré un contexte politico-social catastrophique. En 1932, Willem Keesom et J.N. van den Ende mettent en évidence un saut de la chaleur spécifique de l'étain lors de sa transition de l'état supraconducteur à l'état résistif en champ magnétique nul, et confirment l'absence de chaleur latente de transition. Dans la foulée, Keesom et Johannes Antonie Kok confirment ce résultat pour le thallium. Peu de temps auparavant, Wander Johannes de Haas et H. Bremer avaient trouvé une discontinuité dans la conductivité thermique à la transition en refroidissant en présence d'un champ magnétique, restée elle aussi mystérieuse. Ces résultats sont dans la continuité de l'anomalie de chaleur spécifique de l'hélium à 2,19 K que Keesom et $\mathrm{M}$. Wolfke avaient déjà trouvée en 1927, et qu'ils avaient interprétée immédiatement comme l'indice d'une transition de phase qu'ils baptisent transition entre hélium I et hélium II.

Cet ensemble de résultats amène Ehrenfest à élaborer sa classification des transitions de phase en fonction de leur degré de non-analycité en 1932-1933. Arend Johan Rutgers, premier thésard d'Ehrenfest à Leyde, partant des considérations de celui-ci sur les transitions du second ordre, les applique à la transition supraconductrice et exprime le saut de chaleur spécifique de l'étain en fonction de la température critique $T_{c}$ et de la dérivée du champ critique en fonction de la température à $\mathrm{T}=\mathrm{T}_{\mathrm{c}}$. L'accord est meilleur que le pourcent. C'est la réponse à la vieille question de Langevin au congrès Solvay : l'état supraconducteur est une phase nouvelle, pas l'extrapolation à zéro de la résistivité électrique.

On va donc pouvoir distinguer entre des électrons qui participent à la conductivité métallique ordinaire et ceux qui sont responsables de la supraconduction. Cornelis Jacobus Gorter, récent thésard de de Haas reprend ce résultat et, pour les cas où l'induction est nulle, montre que l'équation de Rutgers est identique à l'application de la seconde loi de la thermodynamique, bien que la disparition des courants persistants par dissipation semble, à première vue, incompatible avec l'hypothèse d'une transition réversible. La notion de flux gelé devenait douteuse.

Une vieille expérience de Kamerlingh Onnes et Tuyn, considérée comme l'expérience modèle du flux gelé présentée par Keesom à la conférence Solvay de 1924, est remise en cause. De Haas et Josina Jonker (sur le point d'épouser Hendrik B.G. Casimir) à Leyden, Walther Meissner à Berlin, reprennent, chacun de leur côté, une série d'expériences pour mieux comprendre l'hystérésis des transitions en champ magnétique. Max von Laue avait fait remarquer que, dans les expériences de de Haas et J. Voogd, il semblait que les résultats sur des fils monocristallins d'étain étaient affectés par les coefficients démagnétisants. L'équipe de de Haas s'engage donc dans de délicates mesures locales de champ magnétique à l'intérieur d'un barreau quand, de Berlin, arrive un résultat inattendu. Meissner et Ochsenfeld viennent de publier un résultat ainsi décrit peu de temps après par Gorter et Casimir : "Lorsqu'un corps devient supraconducteur en présence d'un champ magnétique, la déformation de la distribution des lignes de champ magnétique à l'extérieur du supraconducteur peut être décrite en supposant que le supraconducteur a une susceptibilité $-1 / 4 \pi d$ (où d est la densité)... » Si l'induction magnétique $\mathbf{B}$ est nulle à l'intérieur d'un supraconducteur, c'est l'exact contraire du flux gelé !

\section{La bonne porte d'entrée}

Si la formule de Rutgers appliquée au saut de chaleur spécifique à la transition fût acceptée d'emblée, il n'en alla pas de même, ni pour l'expérience de Meissner-

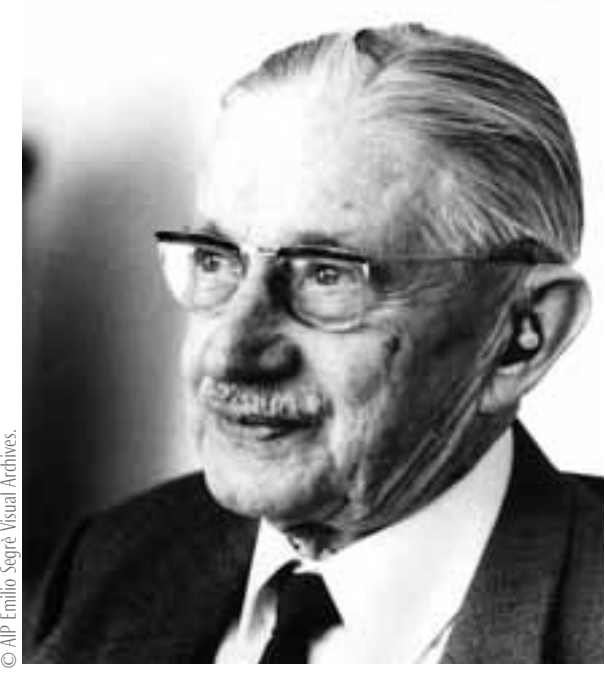

3. Walther Meissner (1882-1974).

Ochsenfeld ni pour son interprétation. Même aujourd'hui, l'expérience n'est pas si facilement reproductible ; de plus, dans le cas d'un tube de plomb creux, Meissner et Ochsenfeld avaient observé que le champ n'était pas diminué à l'intérieur du tube, les deux résultats paraissaient contradictoires. Quant à l'interprétation, Meissner et Ochsenfeld eux-mêmes, suivant le paradigme dominant de la fin des années vingt, voyaient dans l'expérience sur les barreaux massifs d'étain une possible analogie avec le ferromagnétisme.

Le premier à proposer une interprétation radicalement différente fut Gorter, qui envoya immédiatement une note à Nature suggérant que dans l'état supraconducteur $\mathrm{B}$ pouvait toujours valoir zéro. Hypothèse qui avait le mérite de permettre une analyse de la thermodynamique de la transition et d'avancer dans la compréhension du comportement des échantillons supraconducteurs en champ transverse. C'est ce programme que mène à bien la communication de Gorter et Casimir, connue aujourd'hui sous le nom de modèle à deux fluides. Les deux fluides, électrons supraconducteurs et électrons normaux, sont mélangés, le modèle décrit assez correctement la dépendance en température du champ critique. Mais cette proposition ne fit pas l'unanimité. À Kharkov, Shubnikov qui avait séjourné à Leiden et collaboré avec de Haas, reproduisit bientôt le résultat de Meissner sans vaincre le scepticisme de Lev Landau.

En Allemagne, les événements politiques se précipitent, les acteurs de la supraconductivité 


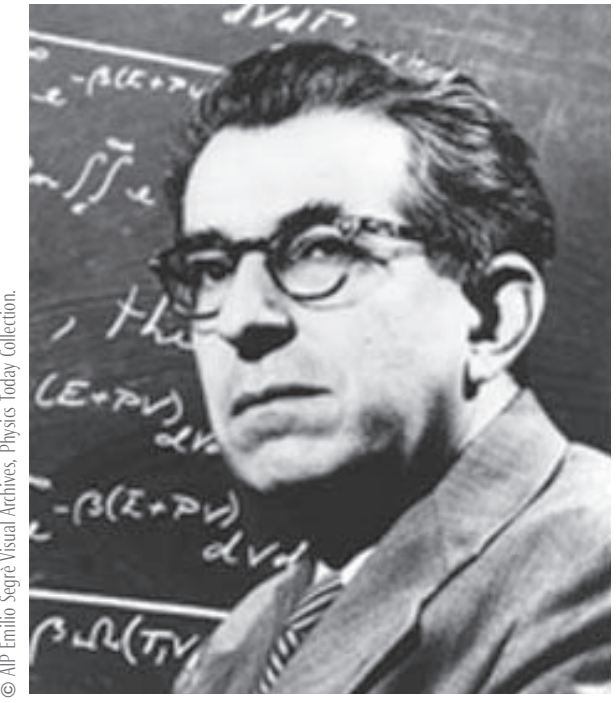

4. Fritz London (1900-1954).

vont eux aussi être pris dans la tourmente. Hitler reçoit les pleins pouvoirs le 24 mars 1933 ; un mois après l'incendie du Reichstag, le 31 mars, l'Académie prussienne est heureuse de se débarrasser d'Einstein ; le 7 avril, la promulgation de la loi pour la restauration des carrières dans la fonction publique (Gesetz zur Wiederherstellung des Berufsbeamtentums, GWB) signifie la révocation des fonctionnaires juifs ou politiquement hostiles. Pour les plus heureux, c'est l'exil et les incertitudes du lendemain.

Fritz London gagne Oxford où l'avenir était bouché puisque Schrödinger, dont il avait été l'assistant, s'y trouve déjà. Au bout de quelques mois, London part pour Paris. Son jeune frère, Heinz, est encore à Breslau pour boucler sa thèse sur l'impédance de surface des supraconducteurs, sous la direction de Franz Simon. Profitant des bourses de deux ans que l'Imperial Chemical Industries avait mises à la disposition de l'Academic Assistance Council pour aider les scientifiques réfugiés, Lindemann invite toute l'équipe de Breslau à Oxford (outre Franz Simon, Kurt Mendelssohn, Nicholas Kurti, Heinz London). Franz Simon emporta dans ses bagages deux liquéfacteurs de poche qu'il avait mis au point, en échange du mobilier de son bureau à l'université, acquis sur ses ressources personnelles. Du jour au lendemain, Oxford devint ainsi un centre de recherches cryogéniques. Un de ces liquéfacteurs sera utilisé pour la première liquéfaction d'hélium en France par Simon et Kurti, au grand électroaimant d'Eugène
Cotton à Meudon-Bellevue. La compagnie Oxford Instruments créée après-guerre par Martin Wood... initialement technicien de Simon, est ainsi une héritière du groupe de Breslau.

Comme Gorter, les frères London étaient convaincus que l'expérience de MeissnerOchsenfeld signifiait que $\mathbf{B}=0$ était la propriété caractéristique de la supraconductivité. Les frères London proposent une description macroscopique, dans laquelle le "supracourant " est seulement déterminé par un champ magnétique. En plus de $\mathbf{B}=0$, ils postulent que le champ électrique $\mathrm{E}$ est nul, et partent d'une seule relation : $c \times \operatorname{rot} \Lambda \mathrm{J}=-\mathrm{B}$, où $\Lambda$ est une grandeur caractéristique du supraconducteur, $c$ est la vitesse de la lumière, $c(\Lambda)^{1 / 2}$ étant la profondeur sur laquelle le champ magnétique est écranté. "Ce qui caractérise le supraconducteur, ce n'est donc pas une valeur particulière de la perméabilité, mais une équation différentielle. " Tous calculs faits, pour un supraconducteur simplement connexe, $\Lambda_{c} \mathrm{~J}=-\mathrm{A}$; le "supracourant " $\mathrm{J}$ qui assure $\mathbf{B}=0$ à l'intérieur du supraconducteur est proportionnel au potentiel vecteur A. «... cette équation exprime la même connexion entre la densité de courant et le champ magnétique que celle qui existe dans un atome diamagnétique isolé [...]. Le supraconducteur en bloc est considéré comme un seul immense atome diamagnétique. "

L'article des deux frères décrivait la supraconductivité sans l'expliquer. Dessinant ce que devrait être selon eux une future "théorie moléculaire " de la supraconductivité, ils supposaient que les électrons devaient être couplés par une interaction à préciser : "Alors, l'état le plus bas des électrons pourrait être séparé par une distance finie de ceux qui sont excités ». Ainsi, dès 1935 c'est, semble-t-il, la première suggestion du gap d'énergie dans les supraconducteurs.

Après qu'il eut donné ses conférences à l'Institut Henri Poincaré en avril 1935 (republiées par Hermann en 2005), Fritz regagna Londres. La Royal Society avait pris l'initiative de réunir tous les acteurs importants de la supraconductivité - sauf les Soviétiques - le 30 mai 1935. London insista sur le fait qu'il avait résolu le dilemme de Bloch-Brillouin. Gorter et Casimir en furent convaincus, mais Meissner répéta qu'il ne voyait dans ses expériences qu'une possible analogie avec le ferromagnétisme.
Aux États-Unis, John Clark Slater et Linus Pauling, partant de l'article de Heitler-London sur la molécule d'hydrogène, l'avaient développé dans une optique pragmatique plus accessible aux chimistes, en mettant l'accent sur les paires électroniques. Heitler et London n'en étaient guère satisfaits, mais London eut en Slater un collègue qui suivit attentivement tous ses travaux et ceux de Gorter et Casimir. Slater, en 1937, publia un article suggérant que la supraconductivité pouvait être décrite par l'application de la théorie des perturbations à la fonction de Bloch pour les électrons dans un métal. Pour un métal, les états excités forment un continuum dont l'état de plus basse énergie est l'état normal (le niveau de Fermi, mais Slater n'emploie pas cette expression). Slater envisageait qu'un petit nombre de ces niveaux puissent avoir leur énergie plus basse que le niveau minimum des électrons normaux, ils constitueraient alors une phase supraconductrice qui ne serait stable qu'à basse température. À cette époque, Slater rencontrait souvent un jeune prodige qui avait quitté l'industrie pétrolière pour faire de la physique. Ensemble, ils discutaient longuement de toutes ces questions et des travaux de London ; c'était John Bardeen. Mais le conflit mondial s'annonçait ; il faudra attendre l'après-guerre pour que reprennent les travaux sur la supraconductivité.

\section{Remerciements}

Les auteurs remercient l'Institut Max Planck d'Histoire des Sciences (Max-Planck-Institut für Wissenschaftsgeschichte) pour la mise à disposition de ses ressources documentaires. GW y ajoute ses remerciements pour l'hospitalité dont il a bénéficié.

\section{En savoir plus}

- J. Matricon et $\mathrm{G}$. Waysand, La guerre du froid Une histoire de la supraconductivité, Le Seuil (1994).

- P.F. Dahl, Superconductivity: Its Historical Roots and Development from Mercury to the Ceramic Oxides, American Institute of Physics (1997).

- F. London, Une conception nouvelle de la supra-conductibilité, conférences faites à l'Institut Poincaré de Paris en 1935, Hermann (1939). Réédition 2005, avec présentation de J. Matricon et G. Waysand.

- K. van der Beek, « Supraconducteurs : la mécanique quantique à grande échelle ", Reflets de la physique 27 (2011-2012), pp. 6-11. 